\title{
MPEG1/perforin-2 mutations in human pulmonary nontuberculous mycobacterial infections
}

\author{
Ryan M. McCormack,, ${ }^{1}$ Eva P. Szymanski, ${ }^{2}$ Amy P. Hsu, ${ }^{2}$ Elena Perez, ${ }^{1}$ Kenneth N. Olivier, ${ }^{3}$ Eva \\ Fisher, ${ }^{1}$ E. Brook Goodhew, ${ }^{1}$ Eckhard R. Podack, ${ }^{1}$ and Steven M. Holland ${ }^{2}$ \\ 'Department of Microbiology and Immunology, University of Miami School of Medicine, Miami, Florida, USA. ${ }^{2}$ Laboratory \\ of Clinical Infectious Diseases, NIAID, NIH, ${ }^{3}$ Cardiovascular and Pulmonary Branch, NHLBI, NIH, Bethesda, Maryland, USA.
}

Perforin-2 is a highly conserved pore-forming protein encoded by macrophage expressed gene 1 (MPEC1). A number of studies have shown that Perforin-2-deficient mice are unable to survive following a bacterial challenge that is nonlethal in WT mice. There is also recent evidence that

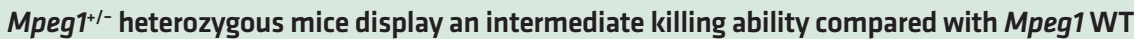
and $M$ peg $1^{-1-}$ mice. Despite these in vivo findings, to date, no perforin-2 deficiencies have been associated with human disease. Here, we report four patients with persistent nontuberculous mycobacterial infection who had heterozygous MPEG1 mutations. In vitro, neutrophils, macrophages, and B cells from these patients were unable to kill Mycobacterium avium as efficiently as normal controls. CRISPR mutagenesis validated the deleterious antibacterial activity of these mutations. These data suggest that perforin-2 haploinsufficiency may contribute to human susceptibility to infections with intracellular bacteria.

Authorship note: RMM and EPS contributed equally to this work. ERP and SMH are co-senior authors. ERP is deceased.

Conflict of interest: The authors have declared that no conflict of interest exists.

Submitted: October 18, 2016

Accepted: March 9, 2017

Published: April 20, 2017

Reference information:

JCI Insight. 2017;2(8):e89635. https:// doi.org/10.1172/jci.insight.89635.

\section{Introduction}

Perforin-2 is a pore-forming protein that is related in structure and function to complement component C9 and Perforin-1. Unlike C9 and Perforin-1 - which are classically involved in killing extracellular bacteria and virus-infected or cancer cells, respectively — perforin-2 is crucial for killing intracellular microbes (1-3). All three proteins share a highly conserved membrane-attack-complex-perforin (MACPF) domain that mediates polymerization and pore formation. Perforin-2 specifically is tethered to the phagosomal membrane by a C-terminal transmembrane domain, such that the N-terminal MACPF and P2 domains are oriented toward the inside of the phagosome. In the resting state, perforin- 2 resides in the endoplasmic reticulum, Golgi, and early endosomal membranes (2). Upon infection, perforin-2 is monoubiquitinated in response to LPS and IFN $\gamma$, and redistributes within the cell to the endosomal/phagosomal bodies that contain phagocytosed bacteria $(1,2,4)$. Following this relocalization, perforin-2 polymerizes and likely refolds to form large clusters of pores that render attacked organisms susceptible to the effects of lysozyme, reactive oxygen species, and nitric oxide, contributing to effective microbial elimination $(1,2)$.

Perforin-2 is encoded by macrophage-expressed gene 1 (MPEG1) (5), a highly conserved, intron-less gene on the antisense strand of chromosome 11. The gene is constitutively expressed in innate immune cells (including macrophages, DCs, NK cells, and neutrophils) and can be significantly upregulated by type I and II interferons, LPS, and bacterial infection (2). Importantly, all cells have the ability to express MPEG1, including barrier cells, such as keratinocytes and mucosal epithelium, and parenchymal cells $(2,6)$. Perforin-2 has been shown to play a role in antibacterial defense in both invertebrate organisms, such as the disk abalone, and in vertebrates such as zebrafish, mouse, and human cells (2, 7-9). Murine macrophages and embryonic fibroblasts restrict intracellular bacterial growth through the agency of perforin- $2(2,6,9)$, whereas $\mathrm{Mpeg}^{-1-}$ cells are unable to kill intracellular pathogens. This killing effect has been reported for different gram-positive, gram-negative, and acid-fast bacteria. To discern whether perforin-2 copy number had any effect on bacterial killing, $\mathrm{Mpeg}^{1^{-+}}$cells were utilized. Interestingly, $\mathrm{Mpeg}^{1^{-1+}}$ cells exhibited only about half the killing activity of WT cells (2), suggesting that perforin-2 levels in the cell are rate limiting for bactericidal activity. This same trend was observed in vivo. Perforin-2-deficient mice rapidly succumbed to challenge with pathogenic bacteria that are nonlethal to WT littermates. Importantly, Mpeg $1^{-1+}$ littermates 
infected with intermediate bacterial inocula had significantly higher death rates than WT mice (2), further supporting that perforin-2 activity is dose sensitive and that Mpeg1 displays haploinsufficiency.

Despite the importance of perforin-2 in bacterial defense, there are no reports of Perforin-2-related pathology in humans. Extrapolating from the murine data, it seemed likely that humans with complete perforin-2 deficiency would be extremely infection susceptible and might not survive infancy. On the other hand, given the striking effects seen in the heterozygous $M p e g 1^{-1+}$ setting, we hypothesized that mutation of one perforin-2 allele and/or a mutation that leads to diminished function without complete loss might be compatible with survival, despite an increased susceptibility to intracellular infection. In order to test this hypothesis, we screened our human pulmonary nontuberculous mycobacterial disease database (10) for MPEG1 mutations.

Mycobacteria are characterized by thick, waxy cell walls (11). In humans, they tend to reside and replicate inside macrophages and possess many methods to resist or evade intracellular killing, making their elimination complex $(12,13)$. Mycobacterial infection triggers IL12 secretion, IFN $\gamma$ production, and macrophage activation leading to killing of intracellular pathogens (14). In contrast to the virulent organism, Mycobacterium tuberculosis, nontuberculous mycobacteria (NTM) are relatively ubiquitous in the environment and only cause disseminated disease in the setting of significant immune compromise. Disseminated NTM infection is most often seen in the context of severe HIV/AIDS, immunosuppressive medication, or genetic defects of the IFN $\gamma / \mathrm{IL} 12$ pathway (15-17). In contrast, pulmonary NTM (PNTM) infection is often seen in the context of cystic fibrosis, primary ciliary dyskinesia, bronchiectasis, or the so-called Lady Windermere syndrome, which is likely a complex polygenic condition facilitated by environmental factors $(10,18-22)$. We previously reported the presence of MPEG1 variants in a cohort of PNTM patients (10). Here, we report those PNTM cases in detail and present functional evidence that human MPEG1/perforin-2 deficiency is likely a contributing factor to pulmonary nontuberculous mycobacterial disease.

\section{Results}

\section{Case Descriptions}

Patient clinical histories and features are presented here and summarized in Supplemental Table 1 (supplemental material available online with this article; https://doi.org/10.1172/jci.insight.89635DS1).

Patient 1. A 70-year-old white woman was first diagnosed with pulmonary Mycobacterium avium complex (MAC) infection at 44 years. She had a distant history of smoking for about 4 years as a young adult. Her medical history included breast cancer, diabetes mellitus type 2, and allergic rhinitis. She had no family history of NTM infections. She had cavitary lung disease that ruptured, leading to a left empyema, lower lobe resection, and Eloesser flap creation at 54 years. She continued to have bronchiectasis, complicated by pulmonary infections with organisms including Pseudomonas, Achromobacter, and Aspergillus species. Whole exome sequence showed heterozygous MPEG1: c.217A>G, p.T73A.

Patient 2. An 86-year-old white woman was first diagnosed with bronchiectasis and MAC at 55 years She had chronic lymphocytic leukemia successfully treated at 61 years and an IgG deficiency, treated with monthly immune globulin. She had no family history of severe infections. Similar to other PNTM patients (23), she had thoracolumbar scoliosis (30 degrees) and pectus excavatum. At age 81 , she developed panuveitis: left eye vitreous fluid grew Brevundimonas species and $\alpha$ hemolytic streptococcus. At the same time, she was found to have a Pseudomonas empyema necessitans, which was surgically drained. Culture-negative endocarditis led to the removal of her pacemaker. She has continued to have pulmonary symptoms and has also had pulmonary infections with $M$. immunogenum. Whole exome sequence showed heterozygous MPEG1: c.946C>T, p.P316S.

Patient 3. A 58-year-old white woman had recurrent pulmonary infections beginning in her 20s and was first diagnosed with MAC and bronchiectasis at 40 years. Her medical history included episodes of oral candidiasis (possibly related to medication use). She continued to have bronchiectasis and pulmonary infections, including Pseudomonas, Achromobacter, and Aspergillus species, and had several episodes of lifethreatening hemoptysis. At 57 years, she had a prolonged hospitalization for lupus nephritis and cerebritis. She continued to have pulmonary symptoms. Whole exome sequence showed heterozygous MPEG1: c.1192C>T, p.Q398X. Of note, her 85-year-old father carried this same truncation but was clinically well.

Patient 4. A white woman diagnosed with MAC and bronchiectasis at 49 years had a 7-year smoking history but quit at 45 years. Through the 7 years since her MAC diagnosis, she had pulmonary infections 


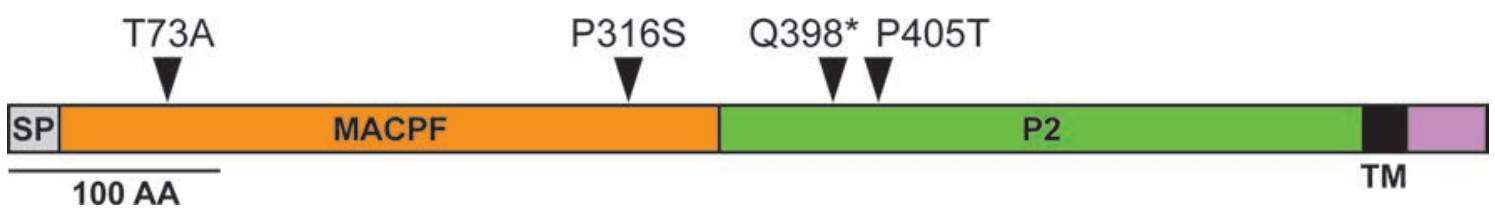

Figure 1. Perforin-2 domains and locations of patient mutations. Colored boxes indicate known domains of the perforin-2 protein. Amino acid ranges for each domain are as follows: signal peptide (SP) from 1-19; membrane-attack-complex-perforin (MACPF) from 30-345; perforin-2 (P2) from 351-653; transmembrane (TM) from 654-674; and cytoplasmic from 675-713. Patient mutations are indicated with arrows.

with Aspergillus fumigatus, Pneumocystis jiroveci, and Bordetella petrii (24). These infections complicated bilateral cavitary lung disease and chronic obstructive pulmonary disease. She succumbed to progressive lung disease at 55 years. Autopsy spleen cultures grew a light amount of B. petrii (24). Whole exome sequence showed heterozygous MPEG1: c.1213C>A, p.P405T.

\section{Patient Mutations}

Out of 70 patients with PNTM disease in our database, four different heterozygous variants in MPEG1 were found in four patients (Supplemental Table 2). These four variants have population frequencies that range from $1.5 \%-0.007 \%$ in European populations of the Exome Aggregation Consortium (ExAC) database. Additionally, the variants are infrequently seen in the homozygous state in ExAC, with incidence ranging from $0.1 \%-0 \%$ across all populations. Looking at alleles for each variant individually, c. $1192 \mathrm{C}>\mathrm{T}$ (p.Q398X) is significantly higher in the PNTM population $(P=8.53 \mathrm{E}-16)$, while the other variants do not reach statistical significance (Supplemental Table 2). When we consider the four MPEG1 variants together, there is not a statistically significant difference in allele frequency between PNTM and control populations $(P=0.7)$. However, these variants are uncommon and may be damaging, especially in the homozygous state. The nucleotide positions of each variant were highly conserved by genomic evolutionary rate profiling (GERP), whereas the amino acid changes predicted ranged from benign to deleterious by Polyphen-2 and Sorting Intolerant from Tolerant (SIFT). We used combined annotation-dependent depletion (CADD), a tool that incorporates multiple types of in silico analyses to calculate an overall Phred score of deleteriousness for each variant in the human genome (25). We found CADD-Phred scores $>15$ for all these variants, indicating that these variants are in at least the top $3 \%$ of deleteriousness across the entire genome, which suggests that they are more likely to have a pathogenic effect. Variant-specific data across each of these measures can be found in Supplemental Table 2. The variants were distributed throughout the gene (Figure 1); three were missense changes. The stop mutation was predicted to truncate the protein just after the MACPF domain, eliminating the P2, transmembrane, and cytoplasmic domains.

\section{Mycobacterial killing was decreased in patient cells with MPEG1 variants}

EBV-transformed B cell lines from each patient with an MPEG1 variant were infected with Mycobacterium avium. Compared with age-matched controls, all of the patient cell lines were significantly less able to control $M$. avium infection, with CFU of $M$. avium increasing in the patient cells over a 24-hour period but decreasing in control cells (Figure 2A). Importantly, MPEG1 mRNA expression levels in patient cells were comparable with control cells (Supplemental Figure 1A). To validate that these findings were not influenced by transformation, fresh peripheral blood was also obtained from Patient 1 (T73A mutation). Both neutrophils and monocyte-derived activated macrophages from this patient demonstrated significant defects in killing $M$. avium compared with both young and age-matched controls (Figure 2, B and C). We were not able to obtain mRNA expression levels of MPEG1 in these cells from Patient 1; however, MPEG1 is constitutively expressed at a high level in human neutrophils and monocyte-derived macrophages (2).

\section{Mycobacterial killing was decreased in cell lines with MPEG1 mutations}

In order to validate that the deleterious effects on $M$. avium killing resulted from these four mutations, CRISPR/Cas9 was used to generate mutations in the THP1 monocyte/macrophage cell line. Heterozygous mutations were individually generated for all four identified MPEG1 mutations. The four mutant cell lines and parental THP1 cell line were infected with Mycobacterium smegmatis under circumstances in which 
the genetic status of the cells was blinded. All MPEG1-mutated cell lines were less-efficient killers of $M$. smegmatis (Figure 3). Again, MPEG1 expression levels in mutant THP1 cell lines were comparable with those in the WT parental cell line (Supplemental Figure 1B). Interestingly, the degree of bacterial killing correlated to the predicted deleteriousness of the MPEG1 mutation, with P316S being the least damaging, followed by T73A, and then P405T and Q398X being the most deleterious to bacterial killing.

\section{Cell lines with MPEG1 mutations are unable to control Salmonella and Staphylococcal infection}

All four patients with $M P E G 1$ variants also had documented pulmonary infections with nonmycobacterial organisms, the most common being Aspergillus species and Pseudomonas aeruginosa. We assessed the ability of mutant THP1 cell lines to control Salmonella enterica spp. typhimurium (S. typhimurium) and Staphylococcus aureus, in an effort to confirm that organisms other than mycobacteria might also have altered killing. All mutant cell lines were significantly less able to control infection with those organisms (Figure 4). Again, killing effectiveness of each mutant cell line correlated with the degree of deleteriousness: p.P316S cells controlled CFU less than WT, while the other mutant cell lines were unable to prevent CFU from increasing. These findings indicate that defects in perforin-2 impair killing ability for multiple pathogenic organisms.

\section{Discussion}

We report the first cases of human MPEG1/perforin-2 deficiency in patients with PNTM infection. Patient peripheral blood phagocytes and EBV-immortalized B cells were less able to control mycobacterial infection in vitro than normal controls. When patient mutations were introduced into a monocyte/macrophage cell line by CRISPR/Cas9, those mutated cell lines were also less able to control in vitro mycobacterial infection.

Perforin-2 has only recently been described, and as such, there are not yet reports of associations between MPEG1 variants and human disease (including tuberculosis). However, the related pore-forming proteins, Perforin-1 and complement C9, are well studied and understood. Recessive mutations in PFN1 (encoding Perforin-1) are associated with poor control of viral infections and lead to the immune disorder familial hemophagocytic lymphohistiocytosis (FHL) (26). Importantly, and relevant to these variants in MPEG1, heterozygous changes in Prf1 in mice and PRF1 in humans are associated with impaired

NK function and contribute to the development of hemo-

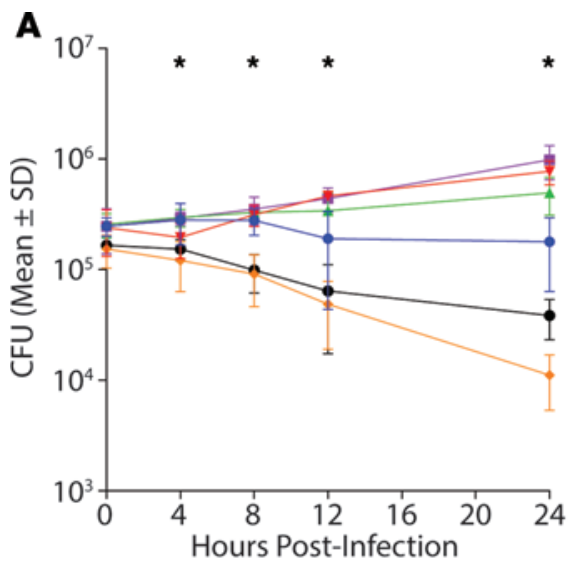

T73A

- p. T73A

p.P316S

p. Q398X

$\approx$ p. P405T

- Age Matched Control \#1

- Age Matched Control \#2

* Young Control

B

C
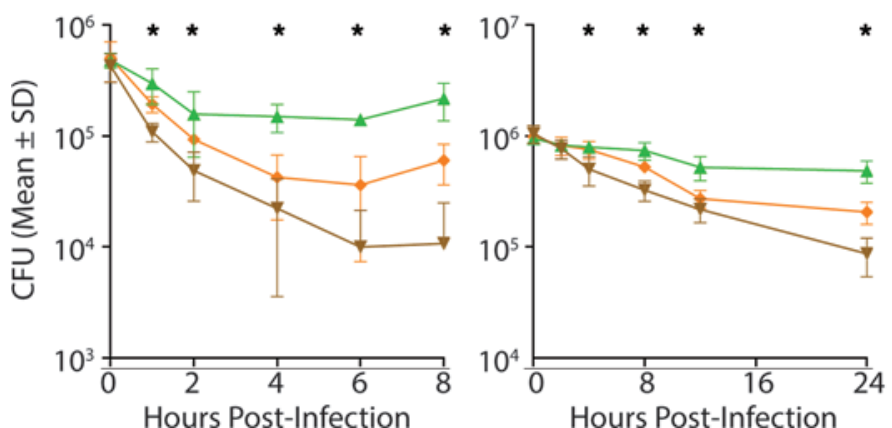

phagocytic lymphohistiocytosis $(\mathrm{HLH})(27,28)$. Mutations have been described in a number of complement pathway genes, including $C 9$, typically leading to bacterial infections including sepsis, meningitis, pneumonia, and respiratory tract infections (29). Some complement mutations are associated with systemic lupus erythematosus (SLE), presumably because of their impaired clearing of complement complexes and antigens (29). In contrast, the mutations in

Figure 2. MPEG1-mutated EBV-transformed B cells, neutrophils, and monocyte-derived macrophages have impaired control of MAC in vitro. (A) Patient EBV-transformed $B$ cell lines are unable to decrease Mycobacterium avium complex (MAC) CFU over time. In comparison, cell lines from age-matched controls show progressive inhibition of MAC CFU. Each time point consists of five biologic replicates and two technical replicates, and the experiment was repeated three times. This graph is representative of four experimental replicates. (B) Neutrophils from Patient 1 (p.T73A) do not inhibit MAC CFU as effectively as controls. (C) Monocyte-derived macrophages from Patient 1 do not inhibit MAC CFU as effectively as controls. Young control is 26 years old; age-matched control \#1 is 57 years old; age-matched control \#2 is 64 years old. $\mathbf{B}$ and $\mathbf{C}$ consist of five biologic replicates and three technical replicates. Graphs are representative of five experimental replicates. Statistical analysis was conducted with one-way ANOVA with Tukey post-hoc test. ${ }^{*} P<0.05$. Detailed statistical breakdown is described in Supplemental Table 6. 


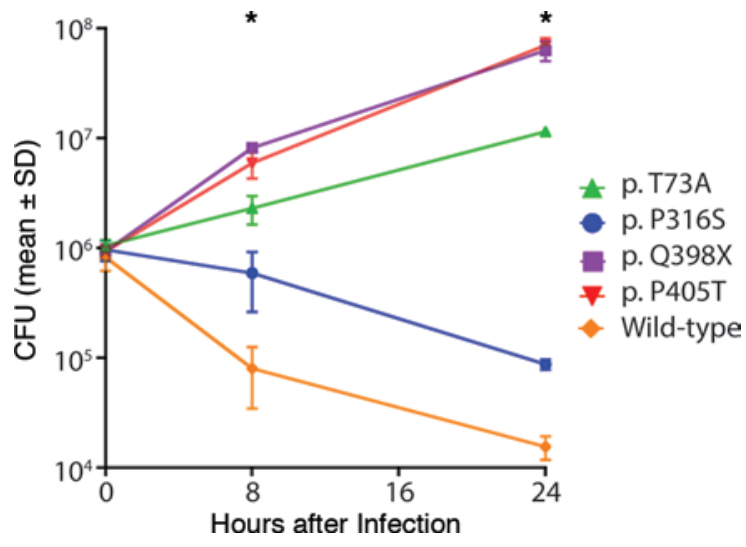

Figure 3. Cell lines with MPEC1/perforin-2 mutations are unable to control Mycobacterium smegmatis infection. Patient MPEG1 mutations were introduced via CRISPR/ Cas9 into THP1 cell lines. WT and mutant cell lines were infected with M. smegmatis, and killing ability was assessed. The WT cell line was able to significantly decrease $M$. smegmatis CFU over time, p.P316S decreased CFU to a lesser extent, and the remaining mutant cell lines were unable to decrease CFU. The experiment was done with five biological replicates and two technical duplicates per replicate, and it was repeated four times. Statistical analysis was conducted by one-way ANOVA with Tukey post-hoc test. ${ }^{*} P<0.05$. Detailed statistical breakdown is described in Supplemental Table 6.

MPEG1 reported here are associated with PNTM and other bacterial and/ or fungal infections. Almost all patients with PNTM infection have bronchiectasis and other organisms in addition to NTM recovered from respiratory specimens. The role of perforin-2 in the control of these other organisms, such as Aspergillus and Pseudomonas, remains to be determined.

The mutations that we identified were heterozygous, suggesting that MPEG1/perforin-2 displays haploinsufficiency. Indeed, Mpeg1 $1^{+/-}$murine cells show an intermediate defect in the killing of M. smegmatis, $M$. avium, and $M$. tuberculosis compared with $M p e g 1^{-1-}$ and WT cells, indicating that perforin-2 gene dosage affects function (2). This in vitro dose-effect was also seen in the phenotypic outcomes of $\mathrm{Mpeg}^{+/-}$mice infected with $S$. typhimurium, which displayed weight loss and survival curves intermediate between those of WT and Mpeg 1-/- mice (2). Therefore, we hypothesize that the heterozygous MPEG1 mutations in our patients rendered one copy of perforin-2 dysfunctional or caused its degradation, leading to reduced functional perforin-2 levels.

With the exception of c.1192C>T (p.Q398X), the frequency of MPEG1 alleles in our PNTM population was not statistically higher than that found in the ExAC population. However, while ExAC excluded subjects with severe pediatric disease, PNTM commonly develops in the fifth or sixth decades, predominantly in females, and markedly more frequently in certain geographic areas (30). Given that ExAC does not contain age or phenotype data, we cannot determine whether any ExAC subjects with MPEG1 variants did or will go on to develop PNTM. Interestingly, looking across the entire MPEG1 gene in ExAC, all variants predicted to have loss of function (including p.Q398X in Patient 3) have allele frequencies of less than $0.02 \%$, and none are seen in the homozygous state (Supplemental Table 3). This supports the hypothesis that complete loss of function of perforin-2 due to homozygous loss-of-function mutations may lead to severe pediatric disease or may not be compatible with life. Conversely, perforin-2 haploinsufficiency is

A

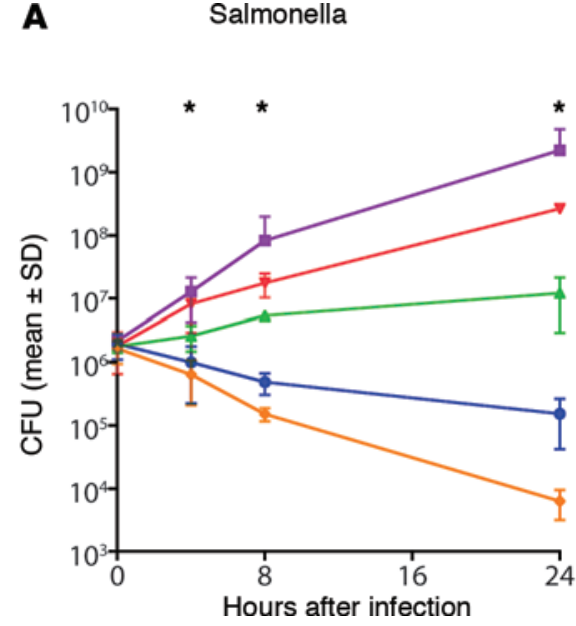

B

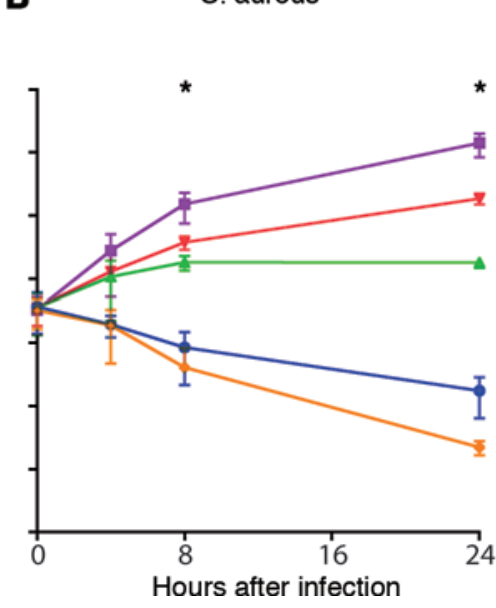

S. aureus

Figure 4. Cell lines with MPEG1/perforin-2 mutations are unable to control S. typhimurium and S. aureus infection. Patient MPEG1 mutations were introduced via CRISPR/Cas9 into THP1 cell lines. WT and mutant cell lines were infected with S. typhimurium (A) and S. aureus (B), and killing ability was assessed. The WT cell line was able to significantly decrease both S. typhimurium and S. aureus CFU over time, p.P316S decreased CFU to a lesser extent, and the remaining mutant cell lines were unable to decrease CFU. The experiment was done with five biological replicates and two technical duplicates per replicate, and it was repeated four times. Statistical analysis was conducted by one-way ANOVA with Tukey post-hoc test. ${ }^{*} P<0.05$. Detailed statistical breakdown is described in Supplemental Table 6. 
relatively rare in adult populations and may account for some degree of other respiratory infections when in the context of other permissive genes or environments.

Our previous exploration of this cohort has suggested that PNTM disease is influenced by multiple gene variants across several functional categories in the context of an aging patient and an infection-permissive environment (10). It seems likely that perforin-2 haploinsufficiency is just one component that may facilitate the development of PNTM disease after reproductive age. Consistent with our polygenic hypothesis, we found variants in CFTR, ciliary, and connective tissue genes in each of our PNTM patients, in addition to the MPEG1 variants (10). The fact that all of these patients were female and symptomatic, while the father of one patient was an older asymptomatic male, raises the possibility that sex influences $M P E G 1 /$ perforin-2 expression or importance, as well. However, our numbers are too small to reach any conclusions.

Our PNTM patients had numerous other organisms recovered from respiratory secretions in addition to NTM, consistent with the fact that polymicrobial flora are common in the bronchiectasis associated with PNTM disease. Sputum samples from Patients 1 and 3 grew Aspergillus, Pseudomonas, and Achromobacter species. Similar to NTM, these organisms are common in the environment and generally do not cause clinical disease in immunocompetent individuals. Patient 1 also had a variant in the $\mathrm{C}$-type lectin gene CLEC4D, which may have contributed to her Aspergillus. Patient 2 did not have additional pulmonary infections, but she did have panuveitis with Brevundimonas, empyema necessitans with Pseudomonas, and an IgG deficiency. Patient 3 had a heterozygous variant in CFTR, which may have affected her mucociliary clearance. Patient 4 had Aspergillus fumigatus, Pneumocystis jiroveci, and Bordetella petrii, in addition to NTM (23). Although we were unable to test these specific organisms in vitro, we did demonstrate that cell lines with $M P E G 1$ variants were unable to control $S$. typhimuri$u m$ and $S$. aureus, suggesting that the consequences of these variants are generalizable to other organisms.

The precise factors in these patients that targeted the lung as the locus of infection remain unclear. Heterozygous MPEG1 mutations may not predispose to severe disseminated disease but may still increase susceptibility to epithelial infection. Perforin-2 expression is inducible in the human U-1752 bronchiolar epithelial cell line, in addition to its constitutive expression in innate immune cells (2). Since the lung is the main portal for airborne environmental entry, the sheer number of exposures over a lifetime might allow a mild genetic defect to eventually become relevant. This is a critical question for further research.

Just as there appears to be a gene dosage effect, there may well be genotype-phenotype correlations that reflect the severity of MPEG1 mutations. Patient 3, who had p.Q398X, which is predicted to cause loss of function and was the least effective at bacterial killing, had recurrent pulmonary infections beginning at age 21 years, was the earliest of our four patients to be diagnosed with PNTM at age 40 years, and developed severe autoimmune disease in her 50s.

Overall, the heterozygous MPEG1 defects that we describe here appear to be clinically relatively mild, which may allow MPEG1/perforin-2 defects to be more common than previously expected. In our study, $6 \%$ of white PNTM patients $(n=4 / 70)$ had a variant in MPEG1 that may have contributed to their pulmonary infection susceptibility (10). Since IFN $\gamma$ upregulates $M P E G 1 /$ perforin-2 and appears to increase MAC killing through a Perforin-2-mediated pathway, these patients might possibly benefit from IFN $\gamma$ therapy. This latter point could provide a missing link in understanding the hitherto elusive mechanism(s) of IFN $\gamma$ 's effect in the immunodeficiency chronic granulomatous disease.

Heterozygous MPEG1/perforin-2 defects were found in four women with PNTM who also had accompanying pulmonary infections. The effect of these variants on susceptibility, therapy, and overall outcome may teach us more about Lady Windermere and other syndromes. MPEG1/perforin-2 appears to be another important mediator of intracellular killing that is under immune regulation.

\section{Methods}

Sequencing. DNA from all patients was sequenced as part of whole exome studies of PNTM susceptibility (dbGaP accession number phs000719.v1.p1), which showed MPEG1 variants (10) that were confirmed by Sanger sequencing (primers available in Supplemental Table 4). Control population frequencies for each variant were obtained from the ExAC database (31). The European population frequency was calculated by combining the European Finnish and European non-Finnish frequencies. GERP scores were added as individual measures of nucleotide evolutionary conservation, while Polyphen- 2 and SIFT scores were added as measures of amino acid change consequence (32-34). The CADD Phred score serves as an overall measure of deleteriousness of each variant compared with the entire genome, in which a score of 10 indicates being in the top $10 \%$ of deleteriousness across the genome, a score of 20 indicates the top 1\%, and so on (25). 
Cells. Peripheral blood mononuclear cells and neutrophils were isolated from healthy donors and PNTM patients. Monocytes were differentiated to macrophages with human macrophage CSF (M-CSF) and then activated with IFN $\gamma$ and LPS overnight as previously described (35). EBV-transformed B cell lines from patients and normal controls were also used. THP1 (TIB-202) and HEK-293 (CRL-1573) cell lines were obtained from the American Type Culture Collection. All cells were cultured at $37^{\circ} \mathrm{C}$ in a humidified atmosphere containing 5\% $\mathrm{CO}_{2}$ following ATCC recommendations for cell culture conditions. M. avium (gift from Timothy Cleary, University of Miami) and M. smegmatis (ATCC 700084) were grown in Middlebrook 7H9 broth. S. typhimurium SL1344 (gift from Jorge Galán, Yale University, New Haven, Connecticut, USA), Staphylococcus aureus CLP148 (MRSA PFGE type USA300) were grown in Luria-Bertani Broth or tryptic soy broth (Sigma-Aldrich) at $37^{\circ} \mathrm{C}$. M. avium was a gift from T. Cleary, University of Miami.

Intracellular bacterial killing assay. Intracellular bacterial killing assays were performed as previously described $(2,9)$. Briefly, cells were infected with $M$. avium or $S$. aureus for 1 hour or $S$. typhimurium for 30 minutes. At the indicated time points, cells were washed with PBS, lysed, diluted, and plated. CFU were determined after colony growth.

Generation of THP1-mutant cell lines. Perforin-2 haploinsufficient cell lines were generated based off of the previously published methods by Zhang and colleagues (36). The guide RNAs (gRNAs) to target the Mpeg1 gene were identified using the http://crispr.mit.edu/ design tool (Supplemental Table 5) and were cloned into pSpCas9n(BB)-2A-Puro (Addgene, PX461) and pSpCas9n(BB)-2A-GFP (Addgene, PX462). THP1 cells were cotransfected with the vectors expressing gRNA and Cas 9 endonuclease, along with donor single-stranded oligonucleotide corresponding to the Mpeg1 WT, T73A, P316S, Q398X, or P405T mutations (Supplemental Table 5) using Nucleofector Kit V (Lonza) according to the recommended protocol. Cells were sorted for $\mathrm{GFP}^{+}$expression and further selected with $1 \mu \mathrm{g} / \mathrm{ml}$ puromycin. Genomic DNA was isolated from each clone for genotyping using QIAamp DNA mini kit (Qiagen). PCRs were performed using the primers surrounding the target site (Supplemental Table 5). The resulting PCR products were screened by SURVEYOR analysis (Integrated DNA Technologies).

Statistics. Frequencies of MPEG1 alleles were compared between the PNTM and ExAC populations using Pearson's chi-squared test with Yates' correction, and a two-tailed $P$ value was generated. $P<0.05$ was considered significant. For bactericidal assays, statistical analysis was conducted using one-way ANOVA with Tukey's post-hoc test. $P<0.05$ was considered significant. All error bars are mean $\pm \mathrm{SD}$. Significant results from the ANOVA statistical testing are listed in Supplemental Table 6.

Study Approval. The human study was reviewed and approved by the National Institute of Allergy and Infectious Diseases Institutional Review Board, Bethesda, Maryland, USA (protocol 01-I-0202). Patients were recruited between 2005 and 2010 at the NIH Clinical Center, Bethesda, Maryland, USA. All patients provided informed consent prior to their participation in the study.

\section{Author contributions}

RMM designed and performed experiments, and revised the manuscript. EPS analyzed whole exome and clinical data, and wrote the manuscript. APH Sanger sequenced samples for clinical validation. EP coordinated the collaboration. KNO treated the patients. EF and EBG assisted with in vitro experiments and generation of cell lines. ERP was the discoverer of Perforin-2, designed experiments, and coordinated the collaboration. SMH conceived the human study and revised the manuscript.

\section{Acknowledgments}

This research was supported by Division of Intramural Research, NIAID, NIH, and the Division of Intramural Research, NHLBI, NIH. The work was supported by grants AI096396, AI0073234, AI110810, AI107062, and NR015649 (to ERP), and by AI106290 and the Lois Pope Life Foundation Developmental Fellowship (to RMM).

The authors would like to thank the ExAC and the groups that provided exome variant data for comparison. A full list of contributing groups can be found at http://exac.broadinstitute.org/about.

Address correspondence to: Steven M. Holland, Laboratory of Clinical Infectious Diseases, Building 10/11N248 MSC 1960, Bethesda, Maryland 20892-1960, USA. Phone: 301.402.7684; E-mail: smh@nih.gov. 
EPS's present address is: Johns Hopkins University School of Medicine, Baltimore, Maryland, USA.

1. McCormack R, de Armas L, Shiratsuchi M, Podack ER. Killing machines: three pore-forming proteins of the immune system. Immunol Res. 2013;57(1-3):268-278.

2. McCormack RM, et al. Perforin-2 is essential for intracellular defense of parenchymal cells and phagocytes against pathogenic bacteria. Elife. 2015;4: e06508.

3. McCormack R, et al. Perforin-2 Protects Host Cells and Mice by Restricting the Vacuole to Cytosol Transitioning of a Bacterial Pathogen. Infect Immun. 2016;84(4):1083-1091.

4. McCormack RM, Lyapichev K, Olsson ML, Podack ER, Munson GP. Enteric pathogens deploy cell cycle inhibiting factors to block the bactericidal activity of Perforin-2. Elife. 2015;4: e06505.

5. Spilsbury K, O’Mara MA, Wu WM, Rowe PB, Symonds G, Takayama Y. Isolation of a novel macrophage-specific gene by differential cDNA analysis. Blood. 1995;85(6):1620-1629.

6. McCormack R, de Armas LR, Shiratsuchi M, Ramos JE, Podack ER. Inhibition of intracellular bacterial replication in fibroblasts is dependent on the perforin-like protein (perforin-2) encoded by macrophage-expressed gene 1. J Innate Immun. 2013;5(2):185-194.

7. Benard EL, et al. Macrophage-expressed perforins mpeg1 and mpeg1.2 have an anti-bacterial function in zebrafish. J Innate Immun. 2015;7(2):136-152.

8. Bathige SD, Umasuthan N, Whang I, Lim BS, Won SH, Lee J. Antibacterial activity and immune responses of a molluscan macrophage expressed gene-1 from disk abalone, Haliotis discus discus. Fish Shellfish Immunol. 2014;39(2):263-272.

9. Fields KA, McCormack R, de Armas LR, Podack ER. Perforin-2 restricts growth of Chlamydia trachomatis in macrophages. Infect Immun. 2013;81(8):3045-3054.

10. Szymanski EP, et al. Pulmonary Nontuberculous Mycobacterial Infection. A Multisystem, Multigenic Disease. Am J Respir Crit Care Med. 2015;192(5):618-628.

11. Puzo G. The carbohydrate- and lipid-containing cell wall of mycobacteria, phenolic glycolipids: structure and immunological properties. Crit Rev Microbiol. 1990;17(4):305-327.

12. Suter E. The multiplication of tubercle bacilli within normal phagocytes in tissue culture. J Exp Med. 1952;96(2):137-150.

13. Stanley SA, Cox JS. Host-pathogen interactions during Mycobacterium tuberculosis infections. Curr Top Microbiol Immunol. 2013;374:211-241.

14. Ramirez-Alejo N, Santos-Argumedo L. Innate defects of the IL-12/IFN- $\gamma$ axis in susceptibility to infections by mycobacteria and salmonella. J Interferon Cytokine Res. 2014;34(5):307-317.

15. Nunn PP, McAdam KP. Mycobacterial infections and AIDS. Br Med Bull. 1988;44(3):801-813.

16. Winthrop KL, Chang E, Yamashita S, Iademarco MF, LoBue PA. Nontuberculous mycobacteria infections and anti-tumor necrosis factor-alpha therapy. Emerging Infect Dis. 2009;15(10):1556-1561.

17. Al-Muhsen S, Casanova JL. The genetic heterogeneity of mendelian susceptibility to mycobacterial diseases. J Allergy Clin Immunol. 2008;122(6):1043-51; quiz 1052.

18. Olivier KN, et al. Nontuberculous mycobacteria. I: multicenter prevalence study in cystic fibrosis. Am J Respir Crit Care Med. 2003;167(6):828-834.

19. Olivier KN, et al. Nontuberculous mycobacteria. II: nested-cohort study of impact on cystic fibrosis lung disease. Am J Respir Crit Care Med. 2003;167(6):835-840.

20. Noone PG, et al. Primary ciliary dyskinesia: diagnostic and phenotypic features. Am J Respir Crit Care Med. 2004;169(4):459-467.

21. Reich JM, Johnson RE. Mycobacterium avium complex pulmonary disease presenting as an isolated lingular or middle lobe pattern. The Lady Windermere syndrome. Chest. 1992;101(6):1605-1609.

22. Chan ED, Iseman MD. Underlying host risk factors for nontuberculous mycobacterial lung disease. Semin Respir Crit Care Med. 2013;34(1):110-123

23. Kim RD, et al. Pulmonary nontuberculous mycobacterial disease: prospective study of a distinct preexisting syndrome. $A m J$ Respir Crit Care Med. 2008;178(10):1066-1074.

24. Zelazny AM, et al. Adaptability and persistence of the emerging pathogen Bordetella petrii. PLoS One. 2013;8(6):e65102.

25. Kircher M, Witten DM, Jain P, O'Roak BJ, Cooper GM, Shendure J. A general framework for estimating the relative pathogenicity of human genetic variants. Nat Genet. 2014;46(3):310-315.

26. Naneh O, Avčin T, Bedina Zavec A. Perforin and Human Diseases. In: Anderluh G, Gilbert R, eds. MACPF/CDC Proteins Agents of Defence, Attack and Invasion. Dordrecht: Springer Netherlands; 2014:221-239.

27. Sepulveda FE, et al. Polygenic mutations in the cytotoxicity pathway increase susceptibility to develop HLH immunopathology in mice. Blood. 2016;127(17):2113-2121.

28. Cohen JI, et al. Late-onset severe chronic active EBV in a patient for five years with mutations in STXBP2 (MUNC18-2) and PRF1 (perforin 1). J Clin Immunol. 2015;35(5):445-448.

29. Skattum L, van Deuren M, van der Poll T, Truedsson L. Complement deficiency states and associated infections. Mol Immunol. 2011;48(14):1643-1655.

30. Adjemian J, Olivier KN, Seitz AE, Holland SM, Prevots DR. Prevalence of nontuberculous mycobacterial lung disease in U.S Medicare beneficiaries. Am J Respir Crit Care Med. 2012;185(8):881-886.

31. Lek M, et al. Analysis of protein-coding genetic variation in 60,706 humans. Nature. 2016;536(7616):285-291.

32. Cooper GM, et al. Distribution and intensity of constraint in mammalian genomic sequence. Genome Res. 2005;15(7):901-913

33. Kumar P, Henikoff S, Ng PC. Predicting the effects of coding non-synonymous variants on protein function using the SIFT algorithm. Nat Protoc. 2009;4(7):1073-1081.

34. Adzhubei IA, et al. A method and server for predicting damaging missense mutations. Nat Methods. 2010;7(4):248-249.

35. Vijayan D. Isolation and differentiation of monocytes-macrophages from human blood. Methods Mol Biol. 2012;844:183-187.

36. Ran FA, Hsu PD, Wright J, Agarwala V, Scott DA, Zhang F. Genome engineering using the CRISPR-Cas9 system. Nat Protoc. 2013;8(11):2281-2308. 